\title{
Computational Studies on the Mechanisms of Nonenzymatic Intramolecular Cyclization of the Glutamine Residues Located at N-Termini Catalyzed by Inorganic Phosphate Species
}

Tomoki Nakayoshi ${ }^{\dagger, \ddagger}$, Koichi Kato ${ }^{\dagger, \S}$, Eiji Kurimoto ${ }^{\dagger}$, Akifumi Oda ${ }^{\dagger, \ddagger, \oplus, *}$

†Graduate School of Pharmacy, Meijo University, 150 Yagotoyama, Tempaku-ku, Nagoya, Aichi 468-8503, Japan

\$nstitute of Medical, Pharmaceutical and Health Sciences, Kanazawa University, Kakuma-machi, Kanazawa, Ishikawa 920-1192, Japan

§Department of Pharmacy, Kinjo Gakuin University, 2-1723 Omori, Moriyama-ku, Nagoya, Aichi 463-8521, Japan

IInstitute for Protein Research, Osaka University, 3-2 Yamadaoka, Suita, Osaka 5650871, Japan

*Corresponding author: oda@meijo-u.ac.jp 
Table S1. B3LYP and MP2 total energies, zero-point energies (ZPEs), Gibbs energies, and thermal corrected energies of optimized geometries. B3LYP total energies (i.e., nuclear repulsion plus electronic energies), ZPEs, and Gibbs energies were calculated by B3LYP/6-31+G(d,p). MP2 total energies were calculated by MP2/6-311+G(d,p)//B3LYP/6-31+G(d,p). Thermal corrected energies were obtained by adding MP2 total energies, ZPEs, and Gibbs energy.

\begin{tabular}{cccccc}
\hline & B3LYP total energy & MP2 total energy & Zero-point energy & Gibbs energy & $\begin{array}{c}\text { Thermal corrected } \\
\text { energy }\end{array}$ \\
\hline RC & -1195.031209 & -1192.531244 & 0.244183 & -0.049599 & -1192.336660 \\
TS1 & -1195.008841 & -1192.512390 & 0.244757 & -0.046508 & -1192.314141 \\
IS1 & -1195.010500 & -1192.517971 & 0.245476 & -0.045979 & -1192.318474 \\
TS2 & -1195.009202 & -1192.517203 & 0.241875 & -0.045356 & -1192.320684 \\
TH & -1195.021123 & -1192.532680 & 0.246413 & -0.046256 & -1192.332523 \\
TS3 & -1195.004574 & -1192.513582 & 0.241301 & -0.045851 & -1192.318132 \\
IS2 & -1195.005482 & -1192.514481 & 0.245034 & -0.046505 & -1192.315952 \\
TS4 & -1194.997642 & -1192.500736 & 0.243639 & -0.047642 & -1192.304739 \\
PC & -1195.041510 & -1192.540565 & 0.241105 & -0.052984 & -1192.352444 \\
\hline
\end{tabular}


Table S2. Cartesian coordinates of RC.

\begin{tabular}{|c|c|c|c|}
\hline Atoms & $x$ & $y$ & $z$ \\
\hline $\mathrm{N}$ & -0.000055 & 0.000139 & 0.000099 \\
\hline $\mathrm{H}$ & -0.000112 & 0.000272 & 1.018731 \\
\hline $\mathrm{H}$ & 1.886000 & 0.000412 & -0.137801 \\
\hline $\mathrm{C}$ & -1.258993 & -0.602891 & -0.456210 \\
\hline $\mathrm{H}$ & -2.108956 & 0.073972 & -0.263249 \\
\hline $\mathrm{C}$ & -1.254304 & -0.861066 & -1.977010 \\
\hline $\mathrm{C}$ & -1.548202 & -1.844153 & 0.413040 \\
\hline $\mathrm{H}$ & -2.263772 & -1.145173 & -2.291444 \\
\hline $\mathrm{H}$ & -1.049144 & 0.089169 & -2.480627 \\
\hline $\mathrm{C}$ & -0.259237 & -1.916757 & -2.487252 \\
\hline $\mathrm{O}$ & -0.932876 & -2.044899 & 1.470860 \\
\hline $\mathrm{H}$ & -0.230063 & -2.791668 & -1.830344 \\
\hline $\mathrm{H}$ & -0.606422 & -2.277488 & -3.463836 \\
\hline $\mathrm{C}$ & 1.143333 & -1.386427 & -2.731909 \\
\hline $\mathrm{N}$ & -2.540371 & -2.661213 & 0.000304 \\
\hline $\mathrm{C}$ & -2.977225 & -3.812723 & 0.781583 \\
\hline $\mathrm{H}$ & -3.038042 & -2.447813 & -0.851058 \\
\hline $\mathrm{H}$ & -2.156648 & -4.523030 & 0.918341 \\
\hline $\mathrm{H}$ & -3.788445 & -4.305357 & 0.245072 \\
\hline $\mathrm{H}$ & -3.335408 & -3.503464 & 1.768404 \\
\hline $\mathrm{P}$ & 2.962864 & 1.808016 & -0.801202 \\
\hline $\mathrm{O}$ & 3.319014 & 1.412819 & -2.359864 \\
\hline $\mathrm{O}$ & 4.197941 & 2.480233 & -0.241329 \\
\hline $\mathrm{O}$ & 1.610166 & 2.506785 & -0.742809 \\
\hline $\mathrm{H}$ & 0.066564 & 0.973172 & -0.306785 \\
\hline $\mathrm{O}$ & 2.815833 & 0.335660 & -0.073677 \\
\hline $\mathrm{H}$ & 2.626227 & 0.829664 & -2.752469 \\
\hline $\mathrm{O}$ & 1.333671 & -0.267695 & -3.245681 \\
\hline $\mathrm{N}$ & 2.161413 & -2.214737 & -2.430635 \\
\hline $\mathrm{H}$ & 2.010163 & -3.103743 & -1.977640 \\
\hline $\mathrm{H}$ & 3.114179 & -1.925139 & -2.604826 \\
\hline
\end{tabular}


Table S3. Cartesian coordinates of TS1.

\begin{tabular}{|c|c|c|c|}
\hline Atoms & $x$ & $y$ & $z$ \\
\hline $\mathrm{N}$ & 0.000039 & -0.000356 & 0.000665 \\
\hline $\mathrm{H}$ & -0.000075 & -0.000633 & 1.023781 \\
\hline $\mathrm{H}$ & 2.971766 & -0.000301 & 0.476466 \\
\hline $\mathrm{C}$ & -1.379825 & -0.170820 & -0.473268 \\
\hline $\mathrm{H}$ & -1.608818 & 0.586240 & -1.227021 \\
\hline $\mathrm{C}$ & -1.473006 & -1.604133 & -1.105083 \\
\hline $\mathrm{C}$ & -2.347266 & -0.014895 & 0.709672 \\
\hline $\mathrm{H}$ & -2.428289 & -2.082697 & -0.874472 \\
\hline $\mathrm{H}$ & -1.396671 & -1.521957 & -2.192209 \\
\hline $\mathrm{C}$ & -0.284626 & -2.393705 & -0.557052 \\
\hline $\mathrm{O}$ & -1.957910 & -0.163070 & 1.878684 \\
\hline $\mathrm{H}$ & -0.448439 & -2.666005 & 0.491346 \\
\hline $\mathrm{H}$ & -0.113117 & -3.314212 & -1.124193 \\
\hline $\mathrm{C}$ & 0.952589 & -1.498877 & -0.694414 \\
\hline $\mathrm{N}$ & -3.626369 & 0.256971 & 0.393516 \\
\hline $\mathrm{C}$ & -4.679344 & 0.365100 & 1.398106 \\
\hline $\mathrm{H}$ & -3.872474 & 0.392040 & -0.577235 \\
\hline $\mathrm{H}$ & -4.782570 & -0.570700 & 1.955109 \\
\hline $\mathrm{H}$ & -5.618522 & 0.583784 & 0.890142 \\
\hline $\mathrm{H}$ & -4.459321 & 1.169714 & 2.105780 \\
\hline $\mathrm{P}$ & 3.115221 & 1.713708 & -0.922642 \\
\hline $\mathrm{O}$ & 3.222125 & 0.639763 & -2.150208 \\
\hline $\mathrm{O}$ & 4.187991 & 2.763151 & -1.106140 \\
\hline $\mathrm{O}$ & 1.655806 & 2.152740 & -0.774728 \\
\hline $\mathrm{H}$ & 0.491846 & 0.847923 & -0.341646 \\
\hline $\mathrm{O}$ & 3.527646 & 0.817630 & 0.399433 \\
\hline $\mathrm{H}$ & 2.515520 & -0.074726 & -2.072402 \\
\hline $\mathrm{O}$ & 1.344339 & -1.203006 & -1.870219 \\
\hline $\mathrm{N}$ & 2.006725 & -1.623871 & 0.293400 \\
\hline $\mathrm{H}$ & 1.699898 & -2.070921 & 1.154268 \\
\hline $\mathrm{H}$ & 2.760097 & -2.178397 & -0.108071 \\
\hline
\end{tabular}


Table S4. Cartesian coordinates of IS1.

\begin{tabular}{|c|c|c|c|}
\hline Atoms & $x$ & $y$ & $z$ \\
\hline $\mathrm{N}$ & 0.000003 & 0.000027 & 0.000016 \\
\hline $\mathrm{H}$ & -0.000006 & 0.000168 & 1.028625 \\
\hline $\mathrm{H}$ & 3.018532 & -0.000028 & 0.258667 \\
\hline $\mathrm{C}$ & -1.419525 & -0.058043 & -0.431666 \\
\hline $\mathrm{H}$ & -1.562884 & 0.621100 & -1.273443 \\
\hline $\mathrm{C}$ & -1.674682 & -1.544754 & -0.860180 \\
\hline $\mathrm{C}$ & -2.298594 & 0.370767 & 0.749941 \\
\hline $\mathrm{H}$ & -2.598755 & -1.932011 & -0.424261 \\
\hline $\mathrm{H}$ & -1.771603 & -1.589960 & -1.946620 \\
\hline $\mathrm{C}$ & -0.433120 & -2.314333 & -0.393424 \\
\hline $\mathrm{O}$ & -1.870705 & 0.299749 & 1.913009 \\
\hline $\mathrm{H}$ & -0.525325 & -2.596154 & 0.662004 \\
\hline $\mathrm{H}$ & -0.253145 & -3.219599 & -0.977665 \\
\hline $\mathrm{C}$ & 0.733267 & -1.322428 & -0.598135 \\
\hline $\mathrm{N}$ & -3.540728 & 0.777803 & 0.443542 \\
\hline $\mathrm{C}$ & -4.520920 & 1.144079 & 1.461629 \\
\hline $\mathrm{H}$ & -3.809780 & 0.840671 & -0.528759 \\
\hline $\mathrm{H}$ & -4.684588 & 0.314093 & 2.154372 \\
\hline $\mathrm{H}$ & -5.459731 & 1.385370 & 0.963748 \\
\hline $\mathrm{H}$ & -4.183037 & 2.014589 & 2.031543 \\
\hline $\mathrm{P}$ & 2.953070 & 1.688049 & -1.157163 \\
\hline $\mathrm{O}$ & 2.853124 & 0.623185 & -2.376695 \\
\hline $\mathrm{O}$ & 3.951651 & 2.770373 & -1.499326 \\
\hline $\mathrm{O}$ & 1.525134 & 2.105074 & -0.758095 \\
\hline $\mathrm{H}$ & 0.529347 & 0.861068 & -0.328844 \\
\hline $\mathrm{O}$ & 3.581519 & 0.803124 & 0.082283 \\
\hline $\mathrm{H}$ & 2.130732 & -0.094147 & -2.178006 \\
\hline $\mathrm{O}$ & 1.061098 & -1.122017 & -1.856499 \\
\hline $\mathrm{N}$ & 1.914287 & -1.496597 & 0.273823 \\
\hline $\mathrm{H}$ & 1.670872 & -1.864969 & 1.192825 \\
\hline $\mathrm{H}$ & 2.512707 & -2.187615 & -0.175010 \\
\hline
\end{tabular}


Table S5. Cartesian coordinates of TS2.

\begin{tabular}{|c|c|c|c|}
\hline Atoms & $x$ & $y$ & $z$ \\
\hline $\mathrm{N}$ & -0.000011 & -0.000045 & 0.000044 \\
\hline $\mathrm{H}$ & -0.000011 & -0.000088 & 1.029688 \\
\hline $\mathrm{H}$ & 3.072905 & -0.000073 & 0.247667 \\
\hline $\mathrm{C}$ & -1.426991 & -0.014815 & -0.421549 \\
\hline $\mathrm{H}$ & -1.552366 & 0.662756 & -1.267504 \\
\hline $\mathrm{C}$ & -1.731681 & -1.495453 & -0.834582 \\
\hline $\mathrm{C}$ & -2.275939 & 0.455566 & 0.766460 \\
\hline $\mathrm{H}$ & -2.656497 & -1.855635 & -0.378085 \\
\hline $\mathrm{H}$ & -1.849938 & -1.546285 & -1.918463 \\
\hline $\mathrm{C}$ & -0.500736 & -2.292449 & -0.381541 \\
\hline $\mathrm{O}$ & -1.831899 & 0.385642 & 1.923495 \\
\hline $\mathrm{H}$ & -0.591108 & -2.585977 & 0.670281 \\
\hline $\mathrm{H}$ & -0.331836 & -3.191199 & -0.978229 \\
\hline $\mathrm{C}$ & 0.671261 & -1.310388 & -0.564123 \\
\hline $\mathrm{N}$ & -3.508789 & 0.896319 & 0.472242 \\
\hline $\mathrm{C}$ & -4.462884 & 1.304291 & 1.499458 \\
\hline $\mathrm{H}$ & -3.789799 & 0.955339 & -0.496975 \\
\hline $\mathrm{H}$ & -4.643666 & 0.486861 & 2.202759 \\
\hline $\mathrm{H}$ & -5.399980 & 1.570746 & 1.011377 \\
\hline $\mathrm{H}$ & -4.089154 & 2.169025 & 2.055467 \\
\hline $\mathrm{P}$ & 2.889336 & 1.600639 & -1.238155 \\
\hline $\mathrm{O}$ & 2.719049 & 0.478891 & -2.350521 \\
\hline $\mathrm{O}$ & 3.842626 & 2.705508 & -1.651898 \\
\hline $\mathrm{O}$ & 1.482070 & 2.041225 & -0.749755 \\
\hline $\mathrm{H}$ & 0.562738 & 0.871539 & -0.330745 \\
\hline $\mathrm{O}$ & 3.602335 & 0.808485 & 0.036296 \\
\hline $\mathrm{H}$ & 1.869737 & -0.316958 & -2.087736 \\
\hline $\mathrm{O}$ & 0.983805 & -1.138679 & -1.859647 \\
\hline $\mathrm{N}$ & 1.868554 & -1.521136 & 0.261605 \\
\hline $\mathrm{H}$ & 1.638878 & -1.877589 & 1.188420 \\
\hline $\mathrm{H}$ & 2.433614 & -2.231622 & -0.199361 \\
\hline
\end{tabular}


Table S6. Cartesian coordinates of TH.

\begin{tabular}{|c|c|c|c|}
\hline Atoms & $x$ & $y$ & $z$ \\
\hline $\mathrm{N}$ & 0.000094 & -0.000173 & 0.000184 \\
\hline $\mathrm{H}$ & -0.000084 & -0.000475 & 1.024641 \\
\hline $\mathrm{H}$ & 2.986829 & 0.000296 & 0.265113 \\
\hline $\mathrm{C}$ & -1.417913 & -0.068312 & -0.410793 \\
\hline $\mathrm{H}$ & -1.569515 & 0.545378 & -1.303347 \\
\hline $\mathrm{C}$ & -1.728654 & -1.575266 & -0.742651 \\
\hline $\mathrm{C}$ & -2.296789 & 0.465517 & 0.723194 \\
\hline $\mathrm{H}$ & -2.605718 & -1.940655 & -0.201820 \\
\hline $\mathrm{H}$ & -1.932743 & -1.677463 & -1.810884 \\
\hline $\mathrm{C}$ & -0.445168 & -2.321272 & -0.346258 \\
\hline $\mathrm{O}$ & -1.928789 & 0.401246 & 1.907222 \\
\hline $\mathrm{H}$ & -0.493788 & -2.655703 & 0.695331 \\
\hline $\mathrm{H}$ & -0.235565 & -3.188137 & -0.977895 \\
\hline $\mathrm{C}$ & 0.652834 & -1.249294 & -0.477352 \\
\hline $\mathrm{N}$ & -3.491827 & 0.965749 & 0.357116 \\
\hline $\mathrm{C}$ & -4.478428 & 1.436527 & 1.323100 \\
\hline $\mathrm{H}$ & -3.721079 & 1.009735 & -0.626064 \\
\hline $\mathrm{H}$ & -4.772158 & 0.631515 & 2.003292 \\
\hline $\mathrm{H}$ & -5.357012 & 1.781915 & 0.778200 \\
\hline $\mathrm{H}$ & -4.077128 & 2.263765 & 1.915847 \\
\hline $\mathrm{P}$ & 2.946764 & 1.779434 & -1.031974 \\
\hline $\mathrm{O}$ & 2.680932 & 0.866682 & -2.236764 \\
\hline $\mathrm{O}$ & 3.861849 & 2.970104 & -1.184496 \\
\hline $\mathrm{O}$ & 1.499849 & 2.309855 & -0.473648 \\
\hline $\mathrm{H}$ & 0.883238 & 1.530163 & -0.342274 \\
\hline $\mathrm{O}$ & 3.515144 & 0.846259 & 0.191409 \\
\hline $\mathrm{H}$ & 1.620232 & -0.383160 & -1.972987 \\
\hline $\mathrm{O}$ & 0.986096 & -1.147610 & -1.831348 \\
\hline $\mathrm{N}$ & 1.880952 & -1.450993 & 0.306071 \\
\hline $\mathrm{H}$ & 1.664479 & -1.694871 & 1.272111 \\
\hline $\mathrm{H}$ & 2.382419 & -2.244285 & -0.089779 \\
\hline
\end{tabular}


Table S7. Cartesian coordinates of TS3.

\begin{tabular}{|c|c|c|c|}
\hline Atoms & $x$ & $y$ & $z$ \\
\hline $\mathrm{N}$ & -0.000001 & -0.000136 & 0.000272 \\
\hline $\mathrm{H}$ & 0.000005 & -0.000364 & 1.025218 \\
\hline $\mathrm{H}$ & 2.644132 & 0.000050 & 0.148184 \\
\hline $\mathrm{C}$ & -1.367519 & -0.403609 & -0.390721 \\
\hline $\mathrm{H}$ & -1.631812 & 0.080730 & -1.334501 \\
\hline $\mathrm{C}$ & -1.349094 & -1.967593 & -0.582285 \\
\hline $\mathrm{C}$ & -2.349283 & 0.028301 & 0.699386 \\
\hline $\mathrm{H}$ & -2.088788 & -2.465459 & 0.050033 \\
\hline $\mathrm{H}$ & -1.582491 & -2.211111 & -1.621026 \\
\hline $\mathrm{C}$ & 0.089287 & -2.380544 & -0.220804 \\
\hline $\mathrm{O}$ & -1.997838 & 0.098273 & 1.888142 \\
\hline $\mathrm{H}$ & 0.159117 & -2.645589 & 0.840027 \\
\hline $\mathrm{H}$ & 0.459321 & -3.218981 & -0.815206 \\
\hline $\mathrm{C}$ & 0.886762 & -1.093936 & -0.509449 \\
\hline $\mathrm{N}$ & -3.608133 & 0.279589 & 0.294045 \\
\hline $\mathrm{C}$ & -4.682126 & 0.617908 & 1.221570 \\
\hline $\mathrm{H}$ & -3.820449 & 0.231074 & -0.692722 \\
\hline $\mathrm{H}$ & -4.815933 & -0.173735 & 1.964477 \\
\hline $\mathrm{H}$ & -5.604918 & 0.734277 & 0.653216 \\
\hline $\mathrm{H}$ & -4.464253 & 1.553453 & 1.745401 \\
\hline $\mathrm{P}$ & 2.478731 & 2.142589 & -1.277220 \\
\hline $\mathrm{O}$ & 2.171720 & 1.156925 & -2.488471 \\
\hline $\mathrm{O}$ & 3.171353 & 3.415060 & -1.720051 \\
\hline $\mathrm{O}$ & 0.988655 & 2.562945 & -0.700542 \\
\hline $\mathrm{H}$ & 0.521297 & 1.725053 & -0.431738 \\
\hline $\mathrm{O}$ & 3.176344 & 1.332601 & -0.143756 \\
\hline $\mathrm{H}$ & 1.694549 & 0.147403 & -2.145248 \\
\hline $\mathrm{O}$ & 1.197099 & -0.961254 & -1.810162 \\
\hline $\mathrm{N}$ & 2.181329 & -1.005247 & 0.316478 \\
\hline $\mathrm{H}$ & 2.045235 & -1.169226 & 1.315890 \\
\hline $\mathrm{H}$ & 2.821202 & -1.721138 & -0.030485 \\
\hline
\end{tabular}


Table S8. Cartesian coordinates of IS2.

\begin{tabular}{|c|c|c|c|}
\hline Atoms & $x$ & $y$ & $z$ \\
\hline $\mathrm{N}$ & 0.000104 & -0.000594 & 0.001146 \\
\hline $\mathrm{H}$ & 0.000199 & -0.001384 & 1.026101 \\
\hline $\mathrm{H}$ & 2.630956 & 0.000069 & 0.187848 \\
\hline $\mathrm{C}$ & -1.361185 & -0.426443 & -0.386438 \\
\hline $\mathrm{H}$ & -1.628881 & 0.041144 & -1.337750 \\
\hline $\mathrm{C}$ & -1.322784 & -1.993281 & -0.555832 \\
\hline $\mathrm{C}$ & -2.352322 & 0.005877 & 0.694315 \\
\hline $\mathrm{H}$ & -2.056878 & -2.490695 & 0.083572 \\
\hline $\mathrm{H}$ & -1.554127 & -2.253706 & -1.591086 \\
\hline $\mathrm{C}$ & 0.120816 & -2.383783 & -0.190537 \\
\hline $\mathrm{O}$ & -2.012395 & 0.075149 & 1.886577 \\
\hline $\mathrm{H}$ & 0.193906 & -2.628929 & 0.875236 \\
\hline $\mathrm{H}$ & 0.497078 & -3.229936 & -0.770055 \\
\hline $\mathrm{C}$ & 0.904226 & -1.092278 & -0.518206 \\
\hline $\mathrm{N}$ & -3.607543 & 0.258679 & 0.277890 \\
\hline $\mathrm{C}$ & -4.688994 & 0.599561 & 1.195674 \\
\hline $\mathrm{H}$ & -3.810897 & 0.210366 & -0.710739 \\
\hline $\mathrm{H}$ & -4.836803 & -0.194904 & 1.932985 \\
\hline $\mathrm{H}$ & -5.604784 & 0.725948 & 0.618164 \\
\hline $\mathrm{H}$ & -4.469790 & 1.530574 & 1.726957 \\
\hline $\mathrm{P}$ & 2.437314 & 2.234632 & -1.276050 \\
\hline $\mathrm{O}$ & 2.266731 & 1.247602 & -2.551163 \\
\hline $\mathrm{O}$ & 3.039756 & 3.548650 & -1.718355 \\
\hline $\mathrm{O}$ & 0.896045 & 2.511187 & -0.785167 \\
\hline $\mathrm{H}$ & 0.488949 & 1.643238 & -0.482995 \\
\hline $\mathrm{O}$ & 3.148445 & 1.449850 & -0.151038 \\
\hline $\mathrm{H}$ & 1.845714 & 0.344392 & -2.253686 \\
\hline $\mathrm{O}$ & 1.233679 & -0.955395 & -1.789660 \\
\hline $\mathrm{N}$ & 2.202350 & -0.979669 & 0.353145 \\
\hline $\mathrm{H}$ & 2.062675 & -1.138746 & 1.352995 \\
\hline $\mathrm{H}$ & 2.858628 & -1.682937 & 0.011731 \\
\hline
\end{tabular}


Table S9. Cartesian coordinates of TS4.

\begin{tabular}{|c|c|c|c|}
\hline Atoms & $x$ & $y$ & $z$ \\
\hline $\mathrm{N}$ & -0.000072 & 0.000266 & -0.000214 \\
\hline $\mathrm{H}$ & -0.000135 & 0.000525 & 1.022936 \\
\hline $\mathrm{H}$ & 2.595117 & -0.000079 & 1.074655 \\
\hline $\mathrm{C}$ & -1.348624 & -0.475296 & -0.391600 \\
\hline $\mathrm{H}$ & -1.685319 & 0.066834 & -1.279880 \\
\hline $\mathrm{C}$ & -1.221505 & -2.012728 & -0.721519 \\
\hline $\mathrm{C}$ & -2.322693 & -0.218352 & 0.761912 \\
\hline $\mathrm{H}$ & -1.975800 & -2.604948 & -0.198144 \\
\hline $\mathrm{H}$ & -1.363576 & -2.165979 & -1.794094 \\
\hline $\mathrm{C}$ & 0.212654 & -2.386180 & -0.296468 \\
\hline $\mathrm{O}$ & -1.927968 & -0.188241 & 1.938093 \\
\hline $\mathrm{H}$ & 0.236665 & -2.701155 & 0.749058 \\
\hline $\mathrm{H}$ & 0.662294 & -3.163782 & -0.916352 \\
\hline $\mathrm{C}$ & 0.933814 & -1.050701 & -0.473125 \\
\hline $\mathrm{N}$ & -3.614758 & -0.068152 & 0.418365 \\
\hline $\mathrm{C}$ & -4.677555 & 0.098299 & 1.404073 \\
\hline $\mathrm{H}$ & -3.861464 & -0.074595 & -0.561597 \\
\hline $\mathrm{H}$ & -4.720214 & -0.762360 & 2.077996 \\
\hline $\mathrm{H}$ & -5.627041 & 0.185879 & 0.876085 \\
\hline $\mathrm{H}$ & -4.515048 & 1.000467 & 2.001073 \\
\hline $\mathrm{P}$ & 2.530190 & 2.537805 & -0.540580 \\
\hline $\mathrm{O}$ & 2.852289 & 1.601327 & -1.854146 \\
\hline $\mathrm{O}$ & 3.040896 & 3.931184 & -0.834596 \\
\hline $\mathrm{O}$ & 0.889438 & 2.594167 & -0.523837 \\
\hline $\mathrm{H}$ & 0.508386 & 1.683525 & -0.353911 \\
\hline $\mathrm{O}$ & 3.012359 & 1.806118 & 0.712273 \\
\hline $\mathrm{H}$ & 2.451774 & 0.700317 & -1.735863 \\
\hline $\mathrm{O}$ & 1.687173 & -0.824353 & -1.438147 \\
\hline $\mathrm{N}$ & 2.227653 & -0.964478 & 1.138930 \\
\hline $\mathrm{H}$ & 1.919084 & -1.155705 & 2.090567 \\
\hline $\mathrm{H}$ & 2.968470 & -1.621829 & 0.907270 \\
\hline
\end{tabular}


Table S10. Cartesian coordinates of PC.

\begin{tabular}{|c|c|c|c|}
\hline Atoms & $x$ & $y$ & $z$ \\
\hline $\mathrm{N}$ & 0.000248 & -0.000037 & -0.000157 \\
\hline $\mathrm{H}$ & 0.000173 & -0.000030 & 1.034368 \\
\hline $\mathrm{H}$ & 2.097706 & 0.000776 & 2.722553 \\
\hline $\mathrm{C}$ & 0.185682 & -1.191215 & -0.812551 \\
\hline $\mathrm{H}$ & -0.541009 & -1.958225 & -0.528420 \\
\hline $\mathrm{C}$ & -0.083276 & -0.671462 & -2.260615 \\
\hline $\mathrm{C}$ & 1.611526 & -1.755009 & -0.672241 \\
\hline $\mathrm{H}$ & 0.560338 & -1.157970 & -2.996075 \\
\hline $\mathrm{H}$ & -1.120486 & -0.892752 & -2.524630 \\
\hline $\mathrm{C}$ & 0.137151 & 0.849109 & -2.171273 \\
\hline $\mathrm{O}$ & 2.601776 & -1.012591 & -0.600546 \\
\hline $\mathrm{H}$ & 1.138380 & 1.150519 & -2.497731 \\
\hline $\mathrm{H}$ & -0.587183 & 1.432501 & -2.744766 \\
\hline $\mathrm{C}$ & 0.010775 & 1.154131 & -0.688471 \\
\hline $\mathrm{N}$ & 1.704846 & -3.099364 & -0.677590 \\
\hline $\mathrm{C}$ & 2.984563 & -3.798619 & -0.640467 \\
\hline $\mathrm{H}$ & 0.857687 & -3.648949 & -0.710622 \\
\hline $\mathrm{H}$ & 3.608196 & -3.509097 & -1.491280 \\
\hline $\mathrm{H}$ & 2.794118 & -4.870952 & -0.684759 \\
\hline $\mathrm{H}$ & 3.526558 & -3.568958 & 0.281644 \\
\hline $\mathrm{P}$ & -0.587838 & 1.553959 & 3.431017 \\
\hline $\mathrm{O}$ & -0.259056 & 2.818409 & 2.454791 \\
\hline $\mathrm{O}$ & -0.244210 & 1.949910 & 4.844273 \\
\hline $\mathrm{O}$ & -2.242738 & 1.434567 & 3.381162 \\
\hline $\mathrm{H}$ & -2.539974 & 0.971736 & 2.583945 \\
\hline $\mathrm{O}$ & -0.008921 & 0.282482 & 2.810152 \\
\hline $\mathrm{H}$ & -0.189339 & 2.572009 & 1.491814 \\
\hline $\mathrm{O}$ & -0.068398 & 2.294337 & -0.186733 \\
\hline $\mathrm{N}$ & 3.090234 & -0.124158 & 2.509018 \\
\hline $\mathrm{H}$ & 3.137621 & -0.468721 & 1.550671 \\
\hline $\mathrm{H}$ & 3.441488 & -0.864774 & 3.111704 \\
\hline
\end{tabular}

